\title{
EDGARD BAVENCOFFE
}

\section{PPCM de suites de polynômes}

Annales de la faculté des sciences de Toulouse $\sigma^{e}$ série, tome $1, \mathrm{n}^{\circ} 2$ (1992), p. 147-168

<http://www.numdam.org/item?id=AFST_1992_6_1_2_147_0>

(C) Université Paul Sabatier, 1992, tous droits réservés.

L'accès aux archives de la revue «Annales de la faculté des sciences de Toulouse » (http://picard.ups-tlse.fr/ annales/) implique l'accord avec les conditions générales d'utilisation (http://www.numdam.org/conditions). Toute utilisation commerciale ou impression systématique est constitutive d'une infraction pénale. Toute copie ou impression de ce fichier doit contenir la présente mention de copyright.

\section{NumDam}

Article numérisé dans le cadre du programme Numérisation de documents anciens mathématiques http://www.numdam.org/ 


\title{
PPCM de suites de polynômes
}

\author{
EDGARD BAVENCOFFE ${ }^{(1)}$
}

RÉSUMÉ. - On étudie ici la limite $r(\mathbf{P})$, pour $n$ tendant vers l'infini, du rapport entre le degré du PPCM et le degré du produit des $n$ premiers termes de quelques suites $\mathbf{P}=\left(P_{k}(T)\right)$ de polynômes. On s'est intéressé aux suites définies, pour $w$ racine primitive $m$-ième de l'unité, par $P_{k}(T)=$ $T^{k n}-w, P_{k}(T)=T^{k m+q}-w$ et $P_{k}(T)=\left(T^{k(n m+1)}-w\right) /\left(T^{k}-w\right)$. Ce problème est lié à celui de la détermination de la limite du rapport entre les logarithmes du PPCM et du produit des $n$ premiers termes d'une suite récurrente binaire.

Abstract. - In this paper, we are interested in the limit $r(\mathbf{P})$, when $n$ tends to infinity, of the quotient of the LCM and the product of the $n$ first terms in a sequence $\mathbf{P}=\left(P_{k}(T)\right)$ of polynomials. We give this limit for the sequences defined by $P_{k}(T)=T^{k n}-w, P_{k}(T)=$ $\left(T^{k(n m+1)}-w\right) /\left(T^{k}-w\right)$, and $P_{k}(T)=T^{k m+q}-w$ where $w$ is a $m$ th primitiv root of unity. This problem is linked with this to determine the limite of the quotient of the logarithm of LCM and product of the $n$ first terms of a binary recurrent sequence.

\section{Introduction}

$\mathbf{P}$ étant une suite $\left(P_{k}(T) \mid k \geq n_{0}\right)$ de polynômes unitaires et à coefficients dans $\mathbb{C}$, on note respectivement $\Pi(n, T, \mathbf{P})$ et $M(n, T, \mathbf{P})$ le produit et le PPCM des polynômes $\left(P_{k}(T) \mid n_{0} \leq k \leq n\right)$.

On se propose ici d'étudier le quotient $r(n, \mathbf{P})$ du degré de $M(n, T, \mathbf{P})$ par le degré de $\Pi(n, T, \mathbf{P})$ et de montrer que pour certaines suites $\mathbf{P}$, la suite $r(n, \mathbf{P})$ admet une limite $r(\mathbf{P})$ lorsque $n$ tend vers l'infini, et on donne la valeur de celle-ci.

(1) Université de Caen, Dépt. de Mathématiques, Esplanade de la Paix, 14032 Caen Cedex (France) 
Cette question est liée au problème suivant : étant donnée une suite $\mathbf{U}=\left(u(k) \mid k \geq n_{0}\right)$ à valeurs dans $\mathbb{Z}^{*}$, on note respectivement $\Pi(n, \mathbf{U})$ et $M(n, \mathrm{U})$ le produit et le PPCM des nombres $\left(|u(k)| \mid n_{0} \leq k \leq n\right)$, on considère le quotient $r(n, \mathrm{U})$ du logarithme de $M(n, \mathrm{U})$ par le logarithme de $\Pi(n, \mathrm{U})$, et on cherche sous quelles conditions la suite $(r(n, \mathrm{U}))$ admet une limite $r(\mathrm{U})$ lorsque $n$ tend vers l'infini.

Il s'avère que si les suites $\mathbf{U}$ et $\mathbf{P}$ sont associées par la relation $u(k)=$ $Q_{k}(\alpha, \beta)$, où $Q_{k}(X, Y)$ est le polynôme homogène associé à $P_{k}(T)$ et où $\alpha$ et $\beta$ sont deux complexes tels que $u(k)$ soit entier relatif non nul, alors pour quelques suites $\mathbf{P}$ et sous réserve de quelques conditions imposées à $\alpha$ et $\beta$, les suites $r(n, \mathbf{U})$ et $r(n, \mathbf{P})$ convergent et ont même limite.

Cette idée a été utilisée par J.-P. Bézivin [5] pour démontrer que si $\alpha$ et $\beta$ sont les racines d'une équation du second degré à coefficients dans $\mathbb{Z}^{*}$ telle que $\alpha \beta$ ne soit pas nul et telle que $\alpha / \beta$ ne soit pas racine de l'unité, alors pour tout entier $m \geq 2$ et pour les suites $P$ et $U$ définies par :

$$
\begin{aligned}
& P(T)=\frac{T^{m}-1}{T-1}, \quad \mathbf{P}=\left(P\left(T^{k}\right) \mid k \geq 1\right) \\
& u(k)=\frac{\alpha^{m k}-\beta^{m k}}{\alpha^{k}-\beta^{k}}, \quad \mathbf{U}=(u(k) \mid k \geq 1),
\end{aligned}
$$

on a :

$$
r(\mathbf{U})=r(\mathbf{P})=\frac{6 H(m)}{(m-1) L(m) \pi^{2}},
$$

expression dans laquelle on a posé :

$$
\begin{aligned}
& L(m)=\prod_{\substack{p \mid m \\
p \text { premier }}}\left(1-\frac{1}{p^{2}}\right), \quad H(m)=\sum_{\substack{d>1 \\
d \mid m}} \frac{d}{m} \varphi(d) \varphi\left(\frac{m}{d}\right), \\
& \varphi(m)=\text { indicateur d'Euler de } m .
\end{aligned}
$$

Ce théorème généralise des formules analogues obtenues par Matiyasevich et Guy [1], Davis [2], Kiss et Matyas [3] et par Akiyama [4], et concernant la suite définie par $F_{0}=0, F_{1}=1, F_{n+2}=a F_{n+1}+b F_{n}$. Leurs résultats ont été étendus aux quotients de nombres de Lehmer par James P. Jones et Peter Kiss [6]. Dans tous les cas, les démonstrations sont de nature arithmétique. 


\section{Remarques concernant le problème général}

Sans faire une étude complète, on peut s'interroger sur le cas de polynômes de la forme $P\left(T^{n}\right)$. Il est évident que la détermination du PPCM $M(N, T, \mathbf{P})$ repose essentiellement sur l'étude des racines communes à deux termes de la suite $\mathbf{P}$.

Si $P\left(z^{n}\right)=P\left(z^{k}\right)=0$, alors $z^{n}=\alpha$ et $z^{k}=\beta$ sont deux zéros de $P(T)$. Ceci conduit à définir une relation d'équivalence sur l'ensemble des complexes en posant $(\alpha \approx \beta) \Leftrightarrow$ (il existe des entiers $n$ et $k$, et un complexe $z$ tels que $z^{n}=\alpha$ et $z^{k}=\beta$ ). Le polynôme $P(T)$ peut alors être mis sous la forme d'un produit de polynômes $Q_{k}(T)$ tels que pour toute racine $\alpha$ de $Q_{i}(T)$ et toute racine $\beta$ de $Q_{j}(T)$ on ait $(\alpha \approx \beta) \Leftrightarrow(i=j)$. Le PPCM des $P\left(T^{n}\right)$ est alors le produit des PPCM des $Q_{k}\left(T^{n}\right)$.

Notons que, pour cette relation, une racine de l'unité est équivalente à, et seulement à, toutes les racines de l'unité (ce qui conduit à s'intéresser à des polynômes $P(T)$ dont tous les zéros sont des racines de l'unité).

Notons aussi que si $P(T)=T-u$, et si $P\left(T^{n}\right)$ et $P\left(T^{k}\right)$ ont une racine commune $z$, on a $z^{n}=z^{k}=u \Rightarrow z^{n-k}=1$. Dans ce cas, $u$ est racine de l'unité. On en déduit que si $\mathbf{P}$ est la suite $\left(T^{n}-u\right)$, et si $u$ n'est pas racine de l'unité, alors $\Pi(n, T, \mathbf{P})=M(n, T, \mathbf{P})$ et on a donc $r(\mathbf{P})=1$.

\section{Notations}

Pour tous entiers $n \geq 1$ et $m \geq 1$, on pose :

$$
\begin{aligned}
(n, m) & =\text { PGCD } n \text { et de } m \\
\phi(n, s, m, T) & =\prod_{\substack{k=1 \\
(k, n m)=1 \\
k \equiv s \bmod m}}^{n m}\left(T-\operatorname{Exp}\left(\frac{2 \pi i k}{n m}\right)\right) \text { pour }(s, m)=1 \\
\phi_{n}(T) & =\text {-ième polynôme cyclotomique } \\
\varphi(n) & =\text { indicateur d'Euler de } n \\
\Omega_{1}(n) & =\prod_{\substack{p \mid n \\
p \text { premier }}}\left(\frac{p}{p+1}\right), \quad \Omega_{2}(n)=\sum_{\substack{k=1 \\
(k, n)=1}}^{n} \frac{1}{k^{2}} .
\end{aligned}
$$


Edgard Bavencoffe

\section{Résultats}

Dans ce qui suit, $m$ est entier fixé $\geq 1, w$ est la racine $\operatorname{Exp}(2 \pi i / m)$ de l'unité, et $r$ est entier premier avec $m$.

THÉORÈme 1. - $n$ et $N$ étant des entiers $\geq 1$, la suite $\mathbf{P}$ étant définie par la relation $k \geq 1 \Rightarrow P_{k}(T)=T^{n k}-w^{r}$, on $a$ :

$$
M(N, T, \mathbf{P})=\prod_{\substack{a=1 \\(a, m)=1}}^{m} \prod_{\substack{b \mid n \\(b, m)=1}} \prod_{\substack{c=1 \\(b, c)=1}}^{N / a} \phi\left(\frac{n c}{b}, s, m, T\right)
$$

où le paramètre $s$ est défini par abs $\equiv r \bmod m$

$$
r(\mathbf{P})=\frac{6 m \Omega_{1}(n m) \Omega_{2}(m) \Omega_{3}(n, m)}{\varphi(m) \pi^{2}}
$$

où

$$
\Omega_{3}(n, m)=\sum_{\substack{k \mid n \\(a, m)=1}} \frac{\varphi(d)}{k d}, \quad \text { avec } d=\operatorname{PGCD}\left(k, \frac{n}{k}\right)
$$

Corolla IRe 1. $-N$ étant entier $\geq 1$, la suite $\mathbf{P}$ étant définie par la relation $k \geq 1 \Rightarrow P_{k}(T)=T^{k}-w^{r}$, on $a$ :

$$
M(N, T, \mathbf{P})=\prod_{\substack{a=1 \\(a, m)=1}}^{m} \prod_{c=1}^{N / a} \phi(c, s, m, T)
$$

où le paramètre $s$ est défini par as $\equiv r \bmod m$

$$
r(\mathbf{P})=\frac{6 m \Omega_{1}(m) \Omega_{2}(m)}{\varphi(m) \pi^{2}} .
$$

THÉORÈME 2. $-m$ étant $\geq 2, N$ et $d$ étant entiers $\geq 1, q$ et $r$ étant entiers premiers avec $m, q$ étant tel que $1 \leq q \leq m$, la suite $\mathbf{P}$ étant définie par la relation $k \geq 0 \Rightarrow P_{k}(T)=\left(T^{d(k m+q)}-w^{r}\right)$, on $a$ :

$$
\begin{aligned}
M(N, T, \mathbf{P})= & \prod_{\substack{a=1 \\
(a, m)=1}}^{m} H\left(x, a, s, m, T^{d}\right), \\
& -150-
\end{aligned}
$$


PPCM de suites de polynômes

$o \grave{u}$

$$
\begin{cases}q s \equiv a r \bmod m & 1 \leq s \leq m \\ a c \equiv q \bmod m & 1 \leq c \leq m \\ x=\frac{N m-(a c-q)}{m c} & \\ H(x, a, s, m, T)=\prod_{0 \leq b \leq x} \phi(a+b m, s, m, T) . & \end{cases}
$$

La condition $q \leq m$ pouvant être supprimée, on $a$ :

$$
r(\mathbf{P})=\frac{6 m \Omega_{1}(m) \Omega_{2}(m)}{\varphi(m) \pi^{2}} .
$$

Corollaire 2. - Les notations étant celles du théorème 2, $m$ étant égal à 3, 4 ou $6, q$ et $r$ prenant en conséquence les valeurs 1 et $m-1$, on $a$ : $M(N, T, \mathbf{P})=H\left(N, q, r, m, T^{d}\right) H\left(\frac{N-m+q+1}{m-1}, m-q, m-r, m, T^{d}\right)$.

La limite $\boldsymbol{r}(\mathbf{P})$ donnée par (6) prend ici les valeurs suivantes :

\begin{tabular}{c|c|c|c}
$m$ & 3 & 4 & 6 \\
\hline$r(\mathbf{P})$ & $\frac{135}{16 \pi^{2}}$ & $\frac{80}{9 \pi^{2}}$ & $\frac{234}{25 \pi^{2}}$
\end{tabular}

ThÉorìme 3. - $n$ et $N$ étant entiers $\geq 1$, la suite $\mathbf{P}$ étant définie par la relation

$$
k \geq 1 \Rightarrow P_{k}(T)=\frac{T^{k(n m+1)}-w^{r}}{T^{k}-w^{r}}
$$

on $a$ :

$$
M(N, T, \mathbf{P})=\prod_{\substack{a=1 \\(a, m)=1}}^{m} \prod_{\substack{\beta \geq 2 \\ \beta \mid(n m+1)}} \prod_{\substack{c=1 \\(c, b)=1}}^{h} \phi(c \beta, s, m, T)
$$

où les paramètres $b, h$ et $s$ sont définis par:

$$
\left\{\begin{array}{l}
\beta \nmid a \Rightarrow h=N / a \\
\beta \mid a \Rightarrow h=N /(a+m) \\
b \beta=n m+1 \\
a b s \equiv r \bmod m
\end{array}\right.
$$


Edgard Bavencoffe

$$
r(\mathbf{P})=\frac{6 \Omega_{1}(m) \Omega_{1}(n m+1)}{n \varphi(m) \pi^{2}} \sum_{a=1}^{m} \sum_{\beta \geq 2} \frac{\beta \varphi(d)}{k^{2} d}
$$

où

$$
\begin{cases}b \beta=n m+1 & \\ d=(b, \beta) & \text { si } \beta \nmid a \\ k=a & \text { si } \beta \mid a . \\ k=a+m\end{cases}
$$

Corolla IRe 3. $-N$ étant entier $\geq 1, n$ étant $\geq 2$, la suite $\mathbf{P}$ étant définie par la relation

$$
k \geq 1 \Rightarrow P_{k}(T)=\frac{T^{k n}-1}{T^{k}-1},
$$

on $a$ :

$$
\begin{gathered}
M(N, T, \mathbf{P})=\prod_{\substack{d \mid n \\
d<n}} \prod_{\substack{k=1 \\
(k, d)=1}}^{N} \phi_{k n / d}(T) \\
R(\mathbf{P})=\frac{6 n \Omega_{1}(n) \Omega_{4}(n)}{(n-1) \pi^{2}}=\frac{6 H(n)}{(n-1) L(n) \pi^{2}}
\end{gathered}
$$

où

$$
\Omega_{4}(n)=\sum_{\substack{k<n \\ k \mid n}} \frac{\varphi(d)}{k d}, \quad \text { avec } d=\operatorname{PGCD}\left(k, \frac{n}{k}\right)
$$

et où $L(n)$ et $H(n)$ sont définis dans le théorème cité dans l'introduction, et donc $r(\mathbf{P})=$ limite donnée dans [5].

\section{Résultats préliminaires}

Lemme 1. - Pour $x>1$ et pour tous entiers naturels $m$ et $n$, on $a$ :

$$
\sum_{\substack{k \leq x \\(k, n)=1}} \varphi(k m)=\frac{3 m x^{2} \Omega_{1}(m n) \varphi(d)}{\pi^{2} d}+\mathrm{O}(x \log x),
$$

avec $d=(m, n)$. 


\section{Résultats utilisés}

Ce lemme a été démontré par Mertens (théorème 3.7 dans [7]) dans le cas $m=n=1$, par J.-P. Bézivin ([5], lemme 5) lorsque $n>1$, par J.-P. Jones et P. Kiss $([6]$, lemmes $6,7,8)$ lorsque $m$ et $n$ sont premiers entre eux.

Démonstration dans le cas où $(m, n)>1$

On procède par récurrence sur le $\operatorname{PGCD}(m, n)$ et on utilise le lemme 8 de l'article de J.-P. Jones et P. Kiss [6]. On pose :

$$
\begin{aligned}
& A(m, n, x)=\sum_{\substack{k \leq x \\
(k, n)=1}} \varphi(k m) \\
& B(m, n, x)=\frac{3 m x^{2} \Omega_{1}(m n) \varphi(d)}{\pi^{2} d}+\mathrm{O}(x \log x)
\end{aligned}
$$

avec $d=(m, n)$.

Le lemme cité affirme que si $d=1$, alors $A(m, n, x)=B(m, n, x)$.

On suppose la relation démontrée pour $(m, n)<d$. Soit $p$ un facteur premier de $d$. On pose $d=\delta p^{e}, m=a p^{\alpha}$ et $n=b p^{\beta}$ où $p$ ne divise pas le produit $\delta a b$. Les nombres premiers avec $b$ se partagent en nombres premiers avec $n$ et en nombres divisibles par $p$ et premiers avec $\mathrm{b}$ :

$$
\begin{gathered}
A(m, b, x)=\sum_{\substack{k \leq x \\
(k, n)=1}} \varphi(k m)+\sum_{\substack{k p \leq x \\
(k, b)=1}} \varphi(k p m)=A(m, n, x)+A\left(p m, b, \frac{x}{p}\right) \\
(m, b)=(p m, b)=\delta<d \Rightarrow\left\{\begin{array}{l}
A(m, b, x)=B(m, b, x) \\
A\left(p m, b, \frac{x}{p}\right)=B\left(p m, b, \frac{x}{p}\right)
\end{array}\right. \\
A(m, n, x)=B(m, b, x)-B\left(p m, b, \frac{x}{p}\right) \quad \text { et } \quad \Omega_{1}(m n)=\Omega_{1}(m b)=\Omega_{1}(p m b) \\
A(m, n, x)=\frac{3 m x^{2} \Omega_{1}(m n) \varphi(\delta)}{\pi^{2} \delta}\left(1-\frac{1}{p}\right)+\mathrm{O}(x \log x) .
\end{gathered}
$$

Le résultat découle alors de l'égalité

$$
\frac{\varphi(d)}{d}=\left(\frac{p-1}{p}\right)\left(\frac{\varphi(\delta)}{\delta}\right) .
$$




\section{Edgard Bavencoffe}

Lemme 2. - Pour $x>1$ et pour tous entiers naturels $m$ et $n$ premiers entre eux, on $a$ :

$$
\sum_{0 \leq k \leq x} \varphi(k m+n)=\frac{3 m^{2} x^{2} \Omega_{1}(m)}{\pi^{2} \varphi(m)}+\mathrm{O}(x \log (x))
$$

Démonstration

$$
\begin{aligned}
C(m, n, x) & =\sum_{k \leq x} \varphi(k m+n)=\sum_{k \leq x} \sum_{d \mid k m+n} \frac{\mu(d)(k m+n)}{d} \\
& =\sum_{d \leq m x+n} \frac{\mu(d) S(d, x)}{d},
\end{aligned}
$$

avec

$$
S(d, x)=\sum_{\substack{k \leq x \\ d \mid(k m+n)}}(k m+n) \text { et } \mu(d)=\text { fonction de Möbius. }
$$

On note que si $(d, m)>1$, alors la condition $(m, n)=1$ implique que $d \nmid(k m+n)$ et donc $S(d, x)=0$. D'où :

$$
C(m, n, x)=\sum_{\substack{d \leq m x+n \\(\bar{d}, m)=1}} \frac{\mu(d) S(d, x)}{d}
$$

Après des calculs sans grand intérêt, on vérifie que si $(d, m)=1$, on a :

$$
\frac{m x^{2}}{2 d}-\frac{3 m x}{2} \leq S(d, x) \leq \frac{m x^{2}}{2 d}+(m+2 n) x
$$

soit

$$
\begin{gathered}
\left|S(d, x)-\frac{m x^{2}}{2 d}\right| \leq M x, \quad \text { avec } M=\frac{3 m+4 n}{2}, \\
\frac{m x^{2} K(m, n, x)}{2}-M x L(m, n, x) \leq C(m, n, x) \\
C(m, n, x) \leq \frac{m x^{2} K(m, n, x)}{2}+M x L(m, n, x) \\
-154-
\end{gathered}
$$


avec

$$
K(m, n, x)=\sum_{\substack{d \leq m x+n \\(\bar{d}, m)=1}} \frac{\mu(d)}{d^{2}} \text { et } L(m, n, x)=\sum_{\substack{d \leq m x+n \\(\bar{d}, m)=1}} \frac{|\mu(d)|}{d}
$$

Compte tenu de la relation

$$
\prod_{p \text { premier }}\left(1-\frac{1}{p^{2}}\right)=\frac{1}{\zeta(2)}=\frac{6}{\pi^{2}}
$$

on obtient :

$$
\begin{aligned}
K(m, n, x) & =\sum_{(d, m)=1} \frac{\mu(d)}{d^{2}}+\mathrm{O}\left(\frac{1}{x}\right)=\prod_{\substack{p \nmid m \\
p \text { premier }}}\left(1-p^{-2}\right)+\mathrm{O}\left(\frac{1}{x}\right) \\
& =\frac{6}{\pi^{2}} \prod_{\substack{p \mid m \\
p \text { premier }}}\left(1-p^{-2}\right)^{-1}+\mathrm{O}\left(\frac{1}{x}\right) \\
& =\frac{6 m \Omega_{1}(m)}{\pi^{2} \varphi(m)}+\mathrm{O}\left(\frac{1}{x}\right) .
\end{aligned}
$$

La conclusion résulte de :

$$
\begin{gathered}
1 \leq L(m, n, x) \leq \sum_{d=1}^{m x+n} \frac{1}{d} \leq 1+\log (m x+n) \\
\frac{m x^{2} K(m, n, x)}{2}=\frac{3 m^{2} x^{2} \Omega_{1}(m)}{\pi^{2} \varphi(m)}+\mathrm{O}(x) \\
\left|C(m, n, x)-\frac{m x^{2} K(m, n, x)}{2}\right| \leq M x L(m, n, x)=\mathrm{O}(x \log x) .
\end{gathered}
$$

LEMME 3. - $a, b, c, m$ et $s$ étant des entiers naturels tels que :

(1) $(s, m)=1$,

(2) $(a, m)=1$,

(3) $a c \equiv s \bmod m$,

(4) tous les diviseurs premiers de $b$ divisent $m$; 
$w$ étant la racine $\operatorname{Exp}(2 \pi i / m)$ de l'unité, $\phi(n, s, m, T)$ étant le polynôme

$$
\prod_{\substack{1 \leq k \leq n m \\(k, n m)=1 \\ k \equiv s \bmod m}}\left(T-\operatorname{Exp}\left(\frac{2 \pi i k}{n m}\right)\right)
$$

on $a$ :

$$
\phi(a b, s, m, T)=w^{c \varphi(a)} \phi_{a}\left(T^{b} w^{-c}\right) .
$$

\section{Démonstration}

Compte tenu des conditions (1) et (4), la condition d'être premier avec $a b m$ est, pour les $k$ qui sont congrus à $s$ modulo $m$ équivalente à $(k, a)=1$. D'autre part, on peut supposer que $s$ satisfait à $1 \leq s \leq m$ :

$$
\phi(a b, s, m, T)=\prod_{k \in E}\left(T-\operatorname{Exp}\left(\frac{2 \pi i k}{a b m}\right)\right)
$$

$$
\begin{aligned}
& E=(k \mid 1 \leq k \leq a b m,(k, a)=1, k \equiv s \bmod m) \\
& E=(k=s+m(a x+y) \mid 0 \leq x \leq b-1,0 \leq y \leq a-1,(s+m y, a)=1)
\end{aligned}
$$

$$
\begin{aligned}
\phi(a b, s, m, T) & =\prod_{\substack{y=0 \\
(s+m y, a)=1}}^{a-1} \prod_{x=0}^{b-1}\left(T-\operatorname{Exp}\left(\frac{2 \pi i(s+m y)}{a b m}\right) \operatorname{Exp}\left(\frac{2 \pi i x}{b}\right)\right) \\
& =\prod_{\substack{y=0 \\
(s+m y, a)=1}}^{a-1}\left(T^{b}-\operatorname{Exp}\left(\frac{2 \pi i(s+m y)}{a m}\right)\right) .
\end{aligned}
$$

Soit $d$ et $q$ tels que $d m=1+a q$

$$
\begin{gathered}
p|(s+m y, a) \Rightarrow p|(d(s+m y)), a) \Rightarrow p|(d s+y+y a q, a) \Rightarrow p|(d s+y) \\
p|(d s+y, a) \Rightarrow p|(d s+y+a q y, a) \Rightarrow p|(d s+d m y, a) \Rightarrow p|(s+m y)
\end{gathered}
$$

donc

$$
(s+m y, a)=1 \quad \Leftrightarrow \quad(d s+y, a)=1 .
$$

On pose $y=u-d s$ et donc $(s+m y, a)=1 \Leftrightarrow(u, a)=1$

$$
\phi(a b, s, m, T)=\prod_{\substack{u=d s \\(u, a)=1}}^{a-1+d s}\left(T^{b}-\operatorname{Exp}\left(\frac{2 \pi i(s+m u-m d s)}{a m}\right)\right)
$$




\section{PPCM de suites de polynômes}

$$
\begin{gathered}
\frac{s(1-m d)}{a m}=-\frac{q s}{m}, \quad q s \equiv q a c=c(d m-1) \equiv-c \bmod m \\
\operatorname{Exp}\left(\frac{2 \pi i s(1-m d)}{a m}\right)=\operatorname{Exp}\left(\frac{2 \pi i c}{m}\right)=w^{c} \\
\phi(a b, s, m, T)=\prod_{\substack{u=d s \\
(u, a)=1}}^{a-1+d s}\left(T^{b}-w^{c} \operatorname{Exp}\left(\frac{2 \pi i u}{a}\right)\right) .
\end{gathered}
$$

Par changement d'indice $u \rightarrow v \equiv u \bmod a$ et $1 \leq v \leq a$, on obtient

$$
\phi(a b, s, m, T)=\prod_{\substack{v=1 \\(v, a)=1}}^{a}\left(T^{b}-w^{c} \operatorname{Exp}\left(\frac{2 \pi i v}{a}\right)\right)=w^{c \varphi(a)} \phi_{a}\left(T^{b} w^{-c}\right) .
$$

Lemme 4.- $m$ et $r$ étant premiers entre eux, $w$ étant la racine $\operatorname{Exp}(2 \pi i / m)$ de l'unité, pour tout entier $n$ on a la relation :

$$
T^{n}-w^{r}=\prod_{\substack{d=1 \\ d \mid n \\(d, m)=1}}^{n} \phi\left(\frac{n}{d}, s, m, T\right),
$$

où le paramètre $s$ est défini par la congruence $d s \equiv r \bmod m$.

\section{Démonstration}

Ceci est une conséquence du lemme 3 . Il existe des entiers $a$ et $b$, uniques, satisfaisant aux conditions (2) et (4) du lemme 3 et tels que $n=a b$. Les diviseurs de $n$ qui sont premiers avec $m$ sont alors les diviseurs de $a$. Dès lors, le paramètre $c$ qui intervient dans la relation (15) pour l'indice $a / d$ est défini par la congruence $s \equiv a c / d \bmod m$, c'est-à-dire $a c \equiv d s \equiv r \bmod m$. II ne dépend donc pas du diviseur $d$. Il suffit ensuite d'appliquer la relation classique $T^{a}-1=\prod \phi_{d}(T)$ où $d$ parcourt l'ensemble des diviseurs de $a$.

LeMme 5. - $m$ et $s$ étant premiers entre eux, le degré du polynôme $\phi(n, s, m, T)$ est égal à $\varphi(n m) / \varphi(m)$.

\section{Démonstration}

Il existe des entiers $a$ et $b$, uniques, satisfaisant aux conditions (2) et (4) du lemme 3 et tels que $n=a b$. Le degré du polynôme $\phi(n, s, m, T)$ est, 
compte tenu de la relation (15), égal à $b \varphi(a)$. Les diviseurs premiers de $b m$ sont exactement ceux de $m$ et on a donc $\varphi(b m)=b \varphi(m)$. D'où :

$\varphi(n m)=\varphi(a b m)=\varphi(b m) \varphi(a)=b \varphi(m) \varphi(a)=\varphi(m) \times \operatorname{deg}(\phi(n, s, m, T))$.

LEMME 6. - $s_{1}$ et $s_{2}$ étant deux entiers premiers avec $m$, les polynômes $\phi\left(n_{1}, s_{1}, m, T\right)$ et $\phi\left(n_{2}, s_{2}, m, T\right)$ sont soit premiers entre eux, soit identiques. L'identité a lieu si et seulement si $n_{1}=n_{2}$ et $s_{1} \equiv s_{2} \bmod m$.

\section{Démonstration}

Si $\phi\left(n_{1}, s_{1}, m, z\right)=\phi\left(n_{2}, s_{2}, m, z\right)$, alors $z$ est racine primitive $m n_{1}$-ième et $m n_{2}$-ième de l'unité, et donc $n_{1}=n_{2}=n$. Dans ce cas $\operatorname{Exp}\left(2 \pi i s_{1} / m\right)=$ $z^{n}=\operatorname{Exp}\left(2 \pi i s_{2} / m\right)$ et donc $s_{1} \equiv s_{2} \bmod m$, d'où l'identité des deux polynômes s'ils ne sont pas premiers entre eux.

\section{Principe de la démonstration des théorèmes}

$\mathbf{P}$ étant la suite $\left(P_{k}(T)\right)$, on écrit chaque polynôme sous forme de produit de polynômes $\phi(q, s, m, T)$ en utilisant le lemme 4. Le PPCM $M(N, T, \mathbf{P})$ est le produit des polynômes $\phi(q, s, m, T)$ distincts qui apparaissent dans ces produits car, d'après le lemme 6 , ces polynômes sont premiers entre eux ou identiques.

On détermine le degré de $M(N, T, \mathbf{P})$ en utilisant le lemme 5. Une estimation de la valeur asymptotique de ce degré lorsque $N$ tend vers l'infini est obtenue par application des lemmes 1 ou 2. On en déduit la valeur de la limite $r(\mathbf{P})$.

\section{Démonstration du théorème 1}

Construction de $M(N, T, \mathbf{P})$

$$
T^{n k}-w^{r}=\prod_{\substack{d=1 \\ d \mid n k \\(d, m)=1}}^{k n} \phi\left(\frac{n k}{d}, s, m, T\right)
$$

où le paramètre $s$ est défini modulo $m$ par la congruence $d s \equiv r \bmod m$. 
Les polynômes associés à $(k, d)$ et $\left(k^{\prime}, d^{\prime}\right)$ sont premiers entre eux sauf s'ils sont identiques, ce qui se produit si et seulement si $k^{\prime} / d^{\prime}=k / d$ et $s^{\prime} \equiv s \bmod m$, où $s$ et $s^{\prime}$ sont définis par $d^{\prime} s^{\prime} \equiv d s \equiv r \bmod m$.

Afin de faire apparaitre les couples $(k, d)$ associés à un même polynôme, on fait intervenir la fraction irréductible $c / b$ qui est égale à $k / d$. Les indices $k$ et $d$ sont donc définis par $k=c a$ et $d=b a$, où $c$ et $b$ sont premiers entre eux. Le produit $c a$ parcourt l'ensemble 1 à $N$. Le produit $b a$ parcourt l'ensemble des diviseurs de can qui sont premiers avec $m$. Ceci se traduit par les conditions $b \mid n,(b, m)=1$ et $(a, m)=1$.

Les polynômes identiques sont définis pour un même couple $(c, d)$ et pour des coefficients $a$ et $a^{\prime}$ tels que $b a \equiv b a^{\prime} \bmod m$. Le paramètre $a$ est donc défini modulo $m$.

En résumé, on obtient tous les polynômes, et on les obtient une seule fois, en posant $k=c a, d=b a$ avec les conditions $b \mid n,(b, m)=1,(c, b)=1$, $1 \leq a \leq m, 1 \leq c a \leq N$. D'où l'expression (1).

Limite $r(\mathbf{P})$

$$
\text { degré } M(N, T, \mathbf{P})=\sum_{\substack{a \leq m \\(a, m)=1}} \sum_{\substack{b \mid n \\(b, m)=1}} \sum_{\substack{c \leq N / a \\(b, c)=1}} \frac{\varphi(m n c / b)}{(m)} .
$$

D'après le lemme 1 :

$$
\sum_{\substack{c \leq N / a \\(c, b)=1}} \varphi\left(\frac{c m n}{b}\right)=\frac{3 m n N^{2} \Omega_{1}(m n) \varphi(d)}{\pi^{2} b a^{2} d}+\mathrm{O}(N \log N),
$$

avec

$$
d=\operatorname{PGCD}\left(\frac{m n}{b}, b\right)=\operatorname{PGCD}\left(\frac{n}{b}, b\right), \quad \operatorname{car}(b, m)=1
$$

degré $M(N, T, \mathbf{P})=\frac{3 m n N^{2} \Omega_{1}(m n)}{\pi^{2} \varphi(m)} \sum_{\substack{a \leq m \\(a, m)=1}} \frac{1}{a^{2}} \sum_{\substack{b \mid n \\(b, m)=1}} \frac{\varphi(d)}{b d}+\mathrm{O}(N \log N)$, où $d=\left(\frac{n}{b}, b\right)$.

$$
\text { degré } M(N, T, \mathbf{P})=\frac{3 m n N^{2} \Omega_{1}(m n) \Omega_{2}(m) \Omega_{3}(n, m)}{\pi^{2} \varphi(m)}+\mathrm{O}(N \log N),
$$


avec

$$
\Omega_{3}(n, m)=\sum_{\substack{b \mid n \\(k, m)=1}} \frac{\varphi(d)}{k d}, \quad \text { où } d=\operatorname{PGCD}\left(k, \frac{n}{k}\right)
$$

La conclusion résulte de cette estimation de degré $M(N, T, \mathbf{P})$ et de degré $\Pi(N, T, \mathbf{P})=\sum_{1 \leq n \leq N} n k=\frac{n N^{2}}{2}+\mathrm{O}(N)$.

\section{Démonstration du corollaire 1}

Pour $n=1$, la relation (1) se simplifie car l'indice $b$ du second produit prend uniquement la valeur 1 , la condition sur $c$ d'être premier avec $b$ est toujours vérifiée, et le paramètre $s$ ne dépend que de $a$.

Quant à la relation (4), elle n'est que la traduction de (2) et de la remarque que $\Omega_{3}(1, m)=1$.

\section{Démonstration du théorème 2}

Construction de $M(N, T, \mathbf{P})$

$$
T^{d(k m+q)}-w^{r}=\prod_{i} \phi\left(j, s, m, T^{d}\right),
$$

avec

$$
\left\{\begin{array}{l}
1 \leq i \text { et } i \mid n m+q \text { et }(i, m)=1 \\
j \text { est défini par } i j=k m+q \\
s \text { est défini, modulo } m, \operatorname{par} i s \equiv r \bmod m .
\end{array}\right.
$$

La condition $(i, m)=1$ est toujours satisfaite car $(q, m)=1$ et $i \mid(n m+q)$. Le paramètre $s$, défini par $q s \equiv i j s \equiv j r \bmod m$ et $1 \leq s \leq m$, est associé à $j$ indépendamment de $n$ car $(q, m)=1$.

Si deux polynômes $\phi\left(j, s, m, T^{d}\right)$ et $\phi\left(j^{\prime}, s^{\prime}, m, T^{d}\right)$ ont une racine commune $z$, alors $z^{d}$ est racine commune de $\phi(j, s, m, T)$ et de $\phi\left(j^{\prime}, s^{\prime}, m, T\right)$. D'après le lemme 6 , ces polynômes sont alors identiques et $j=j^{\prime}, s \equiv$ $s^{\prime} \bmod m$. Le PPCM $M(N, T, \mathbf{P})$ est donc le produit des polynômes distincts 
$\phi\left(j, s, m, T^{d}\right)$ qui apparaissent dans le développement des $\left(T^{d(k m+q)}-w^{r}\right)$ pour $1 \leq k \leq N$

$$
M(N, T, \mathbf{P})=\prod_{j} \phi\left(j, s, m, T^{d}\right)
$$

avec

$$
\left\{\begin{array}{l}
s \text { est défini, modulo } m, \text { par } q s \equiv j r \bmod m \\
\text { il existe } k \text { tel que } 1 \leq k \leq N \text { et } j \mid(k m+q)
\end{array}\right.
$$

Les diviseurs $j$ d'un nombre de la forme $(k m+q)$ sont premiers avec $m$. Réciproquement, si $j$ est un entier premier avec $m$, il est indice acceptable dans ce produit si son plus petit multiple de la forme $(k m+q)$ est tel que $1 \leq k \leq N$.

Soit donc $j \geq 1$ et premier avec $m$. Cet entier s'écrit $a+b m$ où $a$ et $b$ sont entiers, $1 \leq a \leq m$, et $(a, m)=1$. I existe $c$ unique tel que $1 \leq c \leq m$, et $a c \equiv q \bmod m$. Le plus petit multiple de $j$ qui soit de la forme $k m+q$ avec $k \geq 1$ est $c(a+b m)$. La seule condition est $c(a+b m) \leq N m+q$. Le paramètre $s$ est défini modulo $m$ par la congruence $q s \equiv j r \equiv a r \bmod m$.

D'où l'expression de $M(N, T, \mathbf{P})$ :

$$
M(N, T, \mathbf{P})=\prod_{a, b} \phi\left(a+b m, s, m, T^{d}\right),
$$

avec

$$
\left\{\begin{array}{l}
1 \leq a \leq m \text { et }(a, m)=1 \\
c \text { est défini par } 1 \leq c \leq m, a c \equiv q \bmod m \\
0 \leq b \text { et } c(a+b m) \leq N m+q \\
s \text { est défini, modulo } m, \text { par } q s \equiv a r \bmod m,
\end{array}\right.
$$

résultat qui se traduit immédiatement par la relation (5).

\section{Limite $r(\mathbf{P})$}

Dans l'expression (5) de $M(N, T, \mathbf{P})$, le paramètre $a$ est premier avec $m$ et il est donc de même des paramètres $(a+b m)$ qui interviennent dans $H(x, a, s, m, T)$. Une application immédiate du lemme 3 est que si $(n, m)=(s, m)=1$ alors le degré de $\phi(n, s, m, T)$ est $\varphi(n)$. On déduit que :

$$
\begin{aligned}
(a, m)=1 & \Rightarrow \text { degré } \phi(a+b m, s, m, T)=\varphi(a+b m) \\
& \Rightarrow \text { degré } H(x, a, s, m, T)=\sum_{0 \leq b \leq x} \varphi(a+b m) .
\end{aligned}
$$


On applique le lemme 2. Compte tenu de l'expression (5), on obtient :

$$
\text { degré } M(N, T, \mathbf{P})=\sum_{\substack{a=1 \\(a, m)=1}}^{m} \frac{3 d m^{2} x^{2} \Omega_{1}(m)}{\pi^{2} \varphi(m)}+\mathrm{O}(x \log (x))
$$

avec

$$
\begin{gathered}
\left\{\begin{array}{l}
1 \leq c \leq m, \quad a c \equiv q \bmod m \\
x=\frac{N m+q-a c}{m c},
\end{array}\right. \\
\text { degré } M(N, T, \mathbf{P})=\mathrm{O}(N \log (N))+\frac{3 d N^{2} m^{2} \Omega_{1}(m)}{\pi^{2} \varphi(m)} \sum_{\substack{a=1 \\
(a, m)=1}}^{m} \frac{1}{c^{2}}
\end{gathered}
$$

où $c$ est défini par $1 \leq c \leq m, a c \equiv q \bmod m$.

$a$ et $c$ sont univoquement associés et tous deux parcourent l'ensemble des nombres premiers avec $m$ et inférieurs ou égaux à $m$

$$
\begin{gathered}
\sum_{\substack{a=1 \\
(a, m)=1}}^{m} \frac{1}{c^{2}}=\sum_{\substack{c=1 \\
(c, m)=1}}^{m} \frac{1}{c^{2}}=\Omega_{2}(m) \\
\text { degré } M(N, T, \mathbf{P})=\frac{3 d m^{2} \Omega_{1}(m) \Omega_{2}(m) N^{2}}{\pi^{2} \varphi(m)}+\mathrm{O}(N \log (N)) .
\end{gathered}
$$

La conclusion résulte de cette estimation de degré $M(N, T, \mathbf{P})$ et de

$$
\text { degré } \Pi(N, T, \mathbf{P})=\sum_{0 \leq k \leq N} d(k m+q)=\frac{d m N^{2}}{2}+\mathrm{O}(N) .
$$

Supprimer la condition $q \leq m$ revient à une translation de l'indice $k$ dans la suite $P$ et, donc, est sans incidence sur la valeur asymptotique du degré de $M(N, T, \mathbf{P})$ ou de $\Pi(N, T, \mathbf{P})$.

\section{Démonstration du corollaire 2}

On se place dans le cas où $m=3,4$ ou 6 . Les nombres premiers avec $m$ et inférieurs à $m$ sont 1 et $m-1$. Les paramètres $q, r, a$ et $c$ du théorème 2 prennent donc les valeurs :

$$
\begin{aligned}
a=q & \Rightarrow \quad c=1 \quad s=r \quad x=N \\
a=m-q & \Rightarrow \quad c=m-1 \quad s=m-r \quad x=\frac{N-m+q+1}{m-1} .
\end{aligned}
$$




\section{Démonstration du théorème 3}

Construction de $M(N, T, \mathbf{P})$

$$
Q\left(T^{k}\right)=\frac{T^{k(n m+1)}-w^{r}}{T^{k}-w^{r}}=\frac{\prod \phi(q, s, m, T)}{\prod \phi\left(q^{\prime}, s^{\prime}, m, T\right)}
$$

où les paramètres $q, s, q^{\prime}$ et $s^{\prime}$ sont définis par :

$$
\begin{aligned}
& q=\frac{k(n m+1)}{d} \quad d \mid k(n m+1) \quad(d, m)=1 \quad d s \equiv r \bmod m \\
& q^{\prime}=\frac{k}{d^{\prime}} \quad d^{\prime} \mid k \quad\left(d^{\prime}, m\right)=1 \quad d^{\prime} s^{\prime} \equiv r \bmod m .
\end{aligned}
$$

Dans chacun des produits, un même polynôme $\phi$ n'apparaît qu'une fois. Si un polynôme $\phi\left(q^{\prime}, s^{\prime}, m, T\right)$ apparaît au dénominateur, il est associé à un entier $d^{\prime}$ et il apparaît au numérateur, comme polynôme $\phi(q, s, m, T)$ associé à $d=d^{\prime}(n m+1)$ car pour cette valeur de $d$, on a $q=q^{\prime},(d, m)=1$ et $s \equiv s^{\prime} \bmod m$

$$
\begin{aligned}
Q\left(T^{k}\right) & =\prod_{q \in E(k)} \phi(q, s, m, T) \\
E(k) & =\left(q|q| k(n m+1), d=\frac{k(n m+1)}{q},(d, m)=1,(n m+1) \nmid d\right)
\end{aligned}
$$

$s$ étant défini par $1 \leq s \leq m, d s \equiv r \bmod m$.

$M(N, T, \mathbf{P})=\prod \phi(q, s, m, T)$ où les couples $(q, s)$ sont tels que $1 \leq s \leq m$ et tels qu'il existe $d$ et $k$ satisfaisant à

$$
\left\{\begin{array}{l}
1 \leq k \leq N, \quad d q=k(n m+1), \quad(d, m)=1 \\
(n m+1) \nmid d, \quad d s \equiv r \bmod m .
\end{array}\right.
$$

Ces conditions (7.1) étant satisfaites, il existe un et un seul quadruplet $(x, a, b, c)$ défini par :

$$
\left\{\begin{array}{l}
x=(d, k), \quad k=c x, \quad d=b x \\
a \equiv x \bmod m, \quad 1 \leq a \leq m .
\end{array}\right.
$$


Ce quadruplet vérifie les relations :

$$
\left\{\begin{array}{l}
1 \leq a \leq m, \quad(a, m)=1, \quad a \equiv x \bmod m \\
b \mid(n m+1), \quad(n m+1) \nmid b x \\
(b, c)=1, \quad 1 \leq c x \leq N .
\end{array}\right.
$$

Réciproquement, si $(x, a, b, c)$ satisfait à (7.3), les éléments $q, s, k$ et $d$ sont alors définis par (7.4) et ils satisfont aux conditions (7.3) :

$$
\left\{\begin{array}{l}
b q=c(n m+1), \quad a b s \equiv r \bmod m, \quad 1 \leq s \leq m \\
k=c x, \quad d=b x
\end{array}\right.
$$

Reste la question de l'unicité de l'occurence d'un couple $(q, s)$. Deux couples $(b, c)$ et $\left(b^{\prime}, c^{\prime}\right)$ distincts définissent des valeurs $q$ et $q^{\prime}$ distinctes. Pour un même couple $(b, c)$, et donc pour $q$ fixé, des valeurs $a$ et $a^{\prime}$ distinctes définissent des valeurs $s$ et $s^{\prime}$ distinctes. On obtient donc une et une seule fois tous les couples valides $(q, s)$ en prenant tous les triplets $(a, b, c)$ pour lesquels il existe un $x$ tel que le quadruplet $(a, b, c, x)$ satisfasse aux relations (7.3) et en leur adjoignant un et un seul de ces éléments $x$. Notons que (7.3) $\Rightarrow b \leq n m$. Soit donc un triplet $(a, b, c)$ satisfaisant aux conditions (7.5) extraites des conditions (7.3) et complétées par $b \leq n m$

$$
\left\{\begin{array}{l}
1 \leq a \leq m, \quad(a, m)=1 \\
b \mid(n m+1), \quad b \leq n m, \quad(b, c)=1
\end{array}\right.
$$

Les conditions (7.3) seront satisfaites dès lors que $(n m+1) \nmid b x$ et $1 \leq c x \leq$ $N$.

On définit $\beta$ par $b \beta=n m+1$. La condition que $(n m+1)$ ne divise pas $b x$ est équivalente à $\beta \nmid x$. D'où :

$$
\begin{aligned}
& \beta \nmid a \Rightarrow x=a \text { convient } \\
& \beta \mid a \Rightarrow \beta \nmid(a+m) \operatorname{car}(a, m)=1 \Rightarrow x=a+m \text { convient. }
\end{aligned}
$$

Les couples $(q, s)$ qui satisfont aux conditions (7.1) sont ceux qui sont définis par (7.4), par les triplets $(a, b, c)$ qui vérifient (7.5), par la valeur de $x$ qui vient d'être obtenue, et par la condition (7.6) qui en découle :

$$
\left\{\begin{array}{l}
\beta \text { étant défini par } b \beta=(n m+1) \\
\text { si } \beta \nmid a \text { alors } a c \leq N \\
\text { si } \beta \mid a \text { alors } c(a+m) \leq N .
\end{array}\right.
$$


En résumé, le PPCM $M(N, T, \mathbf{P})$ est le produit des $\phi(q, s, m, T)$ tels que $b q=c(n m+1)$ et $a b s \equiv r \bmod m$, les paramètres $a, b$ et $c$ satisfaisant aux relations $(7.5)$ et $(7.6)$.

Limite $r(\mathbf{P})$

$$
\text { Degré } M(N, P, \mathbf{P})=\sum_{\substack{1 \leq a \leq m \\(a, m)=1}} \sum_{\substack{\beta \geq 2 \\ \beta \mid(n m+1)}} \sum_{\substack{1 \leq c \leq h \\(c, b)=1}} \frac{\varphi(c \beta m)}{\varphi(m)}
$$

où les paramètres $b$ et $h$ sont définis par :

$$
\left\{\begin{array}{l}
\beta \nmid a \Rightarrow h=N / a \\
\beta \mid a \Rightarrow h=N /(a+m) \\
b \beta=n m+1 .
\end{array}\right.
$$

Avec ces définitions : d'après le lemme 1 :

$$
\sum_{\substack{1 \leq c \leq h \\(c, b)=1}} \frac{\varphi(c \beta m)}{\varphi(m)}=\frac{3 \beta m h^{2} \Omega_{1}(\beta m b) \varphi(d)}{\pi^{2} d \varphi(m)}+\mathrm{O}(h \log h),
$$

où $d=(\beta m, b)=(\beta, b)$ car $(b, m)=1$,

$$
\begin{gathered}
b \beta=n m+1 \Rightarrow(b \beta, m)=1 \Rightarrow \Omega_{1}(\beta m b)=\Omega_{1}(m) \Omega_{1}(n m+1) \\
\left\{\begin{array}{l}
h=N / a \text { ou } N /(m+a) \\
1 \leq a \leq m
\end{array} \Rightarrow \mathrm{O}(h \log h)=\mathrm{O}(N \log N) .\right.
\end{gathered}
$$

D'où l'expression du degré de $M(N, T, \mathbf{P})$ :

$$
\text { degré } M(N, T, \mathbf{P})=\frac{n m N^{2} Z(n, m)}{2}+\mathrm{O}(N \log N)
$$

avec

$$
Z(n, m)=\frac{6 \Omega_{1}(m) \Omega_{1}(n m+1)}{n \varphi(m) \pi^{2}} \sum_{a=1}^{m} \sum_{\substack{\beta \geq 2 \\ \beta \mid(n m+1)}} \frac{\beta \varphi(d)}{k^{2} d}
$$

où

$$
\begin{cases}b \beta=n m+1 & d=(b, \beta) \\ k=a & \text { si } \beta \nmid a \\ k=a+m & \text { si } \beta \mid a .\end{cases}
$$




\section{Edgard Bavencoffe}

La condition résulte de cette estimation de degré $M(N, T, \mathbf{P})$ et de

$$
\text { degré } \Pi(N, T, \mathbf{P})=\sum_{1 \leq k \leq N} k n m=\frac{n m N^{2}}{2}+\mathrm{O}(N) .
$$

\section{Démonstration du corollaire 3}

Le corollaire est le théorème 3 pour $m=1$, avec le changement de paramètre $n \rightarrow n-1$. On note que le paramètre $a$ prend uniquement la valeur $a=1$, que $\beta$ est diviseur de $n$ et qu'il ne divise pas $a=1$, qu'en conséquence $h$ est alors égal à $N$, que le paramètre $b$ est défini par $b \beta=n$, que $s=r=1$, et enfin que $\phi(k, 1,1, T)=\phi_{k}(T)$ pour tout entier $k$. D'où l'expression (11) du PPCM.

Quant à la relation (12), elle n'est que l'application de la relation (10) au cas $m \rightarrow 1$ et $n \rightarrow n-1$. On note que le paramètre $a$ ne prend que la valeur $a=1$. D'où :

$$
r(\mathbf{P})=\frac{6 \Omega_{1}(1) \Omega_{1}(n)}{(n-1) \pi^{2}} \sum_{a=1} \sum_{\beta \geq 2} \frac{\beta \varphi(d)}{k^{2} d},
$$

où

$$
b \beta=n, \quad d=(b, \beta), \quad \beta \nmid a \Rightarrow k=1 .
$$

On fait le changement d'indice $\beta \rightarrow k=m / \beta$ pour obtenir la relation (12). Enfin, pour établir l'identité avec la relation obtenue par J.-P. Bezivin dans [5], il suffit de noter que si $d$ est le PGCD des entiers $x$ et $y$, alors $\varphi(d) \varphi(x y)=d \varphi(x) \varphi(y)$.

\section{Remarques et questions ouvertes}

Dans cette partie, on se propose de présenter le lien entre l'étude faite et le problème du PPCM de suites récurrentes binaires.

Soient $a$ et $b$ deux entiers relatifs, premiers entre eux, et les racines $\alpha$ et $\beta$ de l'équation $t^{2}=a t+b$. On suppose que $\alpha \beta$ n'est pas nul, et que $\alpha / \beta$ n'est pas racine de l'unité. Soit $\mathbf{U}$ une suite d'éléments $u(n)$ de $\mathbb{Z}^{*}$ telle que $u(n+2)=a u(n+1)+b u(n)$. 
Ces entiers s'écrivent $u(n)=c\left(\alpha^{n}-w \beta^{n}\right)=c Q\left(\alpha^{n}, \beta^{n}\right)$ où $Q(X, Y)=$ $X-w Y$ est le polynôme homogène associé à $P(T)=T-w$.

Notons que si $w$ est une racine imaginaire de l'unité, alors $w$ est égal à $\pm i, \pm j$ ou $\pm j^{2}$ et $\alpha$ et $\beta$ appartiennent à $\mathbb{Z}[w]$.

La connaissance du PPCM des polynômes $P(T), \ldots, P\left(T^{n}\right)$ renseigne en partie sur le PPCM des nombres $u(1), \ldots, u(n)$. On peut penser que la spécialisation $T \rightarrow \alpha / \beta$ modifie peu la situation et donc que le rapport entre les degrés du PPCM et du produit des $n$ premiers polynômes d'une part, et le rapport entre les logarithmes du PPCM et du produit des $n$ premiers éléments de $U$ d'autre part, convergeront en même temps et vers la même limite (si elle existe) lorsque $n$ tend vers l'infini.

Notons qu'une modification finie de $U$ est sans incidence pour ce problème, et qu'on peut donc décaler les indices. Le terme $w$ est donc défini à un facteur $\alpha^{k} / \beta^{k}$ près. Avec cette convention quant à la définition de $w$, et compte tenu de l'étude ici faite et de l'hypothèse avancée quant au parallèle entre les suites de polynômes et les suites arithmétiques, les questions suivantes se posent immédiatement.

1) Si $w$ n'est pas racine de l'unité, a-t-on $r(\mathbf{U})=1$ ?

Cette question est, à la connaissance de l'auteur, ouverte.

2) Si $w=+1$, a-t-on $r(\mathrm{U})=6 / \pi^{2}$ ?

Réponse : oui (voir [1], [2], [3])

U est la suite de Fibonacci définie par :

$$
u(n)=\frac{\alpha^{n}-\beta^{n}}{\alpha-\beta} .
$$

3) Si $w=-1$, a-t-on $r(\mathrm{U})=8 / \pi^{2}$ ?

Réponse : oui (voir [5])

U est la suite de Lucas définie par :

$$
u(n)=\alpha^{n}+\beta^{n} .
$$

4) Si $w= \pm i, \pm j, \pm j^{2}$, a-t-on pour $r(\mathrm{U})$ les valeurs obtenues dans le corollaire 1 ?

$$
\frac{80}{9 \pi^{2}}, \quad \frac{135}{16 \pi^{2}}, \quad \frac{234}{25 \pi^{2}} \text {. }
$$

Dans un article en cours de rédaction, l'auteur a pu montrer que, pour ces valeurs de $w$, pour $\alpha$ et $\beta$ premiers entre eux dans $\mathbb{Z}[w]$ 
et, pour les suites $\mathbf{P}$ étudiées dans cette note, la suite $\mathbf{U}$ définie par $u(k)=c P(\alpha, \beta)$ (où $c$ est un facteur tel que $u(k) \in \mathbb{Z}^{*}$ ) vérifie $r(\mathbf{U})=r(\mathbf{P})$.

Ceci est en particulier vrai pour la suite $\mathbf{U}$ définie par $u(n)=$ $c\left(\alpha^{n}-w \beta^{n}\right)$. Sous réserve d'une étude plus approfondie, il semble que ceci puisse être étendu à d'autres valeurs de $w$.

Notons que des suites $\mathbf{P}$ et $\mathbf{U}$ associées par $u(n)=P_{n}(x)$ peuvent être telles que $r(\mathbf{U})=r(\mathbf{P})$ sans que le logarithme de $M(N, \mathbf{U})$ soit équivalent au degré de $M(N, T, \mathbf{P})$. Un exemple est donné par $u(n)=\phi_{n}( \pm 1)$. L'étude de la suite $\phi_{n}(x)$, pour $x \in \mathbb{Z}^{*}$ et différent de \pm 1 , se heurte aux mêmes difficultés que celle de la suite $\alpha^{n}-w \beta^{n}$ lorsque $w$ n'est pas racine de l'unité.

Enfin, on peut signaler qu'il ne semble pas qu'il existe une étude du problème polynomial général et, en particulier, des suites définies par $p_{k}(T)=P\left(T^{k}\right)$. De même, l'étude de ce problème dans le cas de plusieurs variables permettrait d'aborder l'étude du PPCM de suites récurrentes de longueur supérieure à 2 .

\section{Bibliographie}

[1] Matiyasevich (Y. V.) et Richard (K. G.) . - A new formula for $\pi$, American Mathematic Monthly 93 (1986), pp. 631-635.

[2] Davis (M.) .- Hilbert's tenth problem is unsolvable, American Mathematic Monthly 80 (1973), pp. 233-269.

[3] Kiss (P.) et Matyas (F.) .- An asymptotic formula for $\pi$, Journal of Number Theory 31 (1989), pp. 255-259.

[4] Aкiyama (S.) . - Lehmer numbers and as asymptotic formula for $\pi$, Journal of Number Theory 36 (1990), pp. 328-331.

[5] Bézivin (J.-P.) . - Plus petit commun multiple des termes consécutifs d'une suite récurrente linéaire,

Collectanea Mathematica 40, $\mathrm{n}^{\circ} 1$ (1989), pp. 1-11.

[6] Jones (J. P.) et KIss (P.) .- An asymptotic formula concerning Lehmer numbers,

à paraître.

[7] Apostol (T. M.) .- Introduction to analytic number theory,

Springer-Verlag (1976). 